\title{
Text Line
}

National Cancer Institute

\section{Source}

National Cancer Institute. Text Line. NCI Thesaurus. Code C49259.

Text consisting of a row of words written across a page or computer screen. 